\title{
Colo-Cystic Duct Fistula: An Unknown Complication of Colonic Diverticular Disease
}

\author{
Lakshmi Kant Pathak $^{\mathrm{a}, \mathrm{c}}$, Vimala Vijayaraghavan ${ }^{\mathrm{b}}$
}

\begin{abstract}
Enterobiliary fistula is a complication of gall stones and gall bladder disease. Diverticular diseases are known for fistulous complication but enterobiliary fistula as a complication is unknown. This case presents a patient with diverticular disease with lower gastro-intestinal bleed who was found to have a colo-cystic duct fistula at surgery.
\end{abstract}

Keywords: Colo-cystic fistula; Diverticulosis; Entero-biliary fistula

\section{Introduction}

Spontaneous enterobiliary fistulas (EBFs) are a complication of biliary disease or a disease of adjacent structures. They are usually associated with gallstones, but have also been reported with peptic ulcer disease, abdominal trauma, Crohn's disease, and malignancies of the biliary tract, bowel, and head of the pancreas [1]. Communication can occur between the gallbladder or biliary system and the stomach, small intestine, or colon. Although the incidence of such fistulas is unknown, autopsy studies estimate it as less than 1\%. Cholecysto-duodenal fistulas are the most frequent $(75 \%)$, cholecysto-colic is the next most common (15\%), and all other types comprise the remaining $10 \%$. The signs and symptoms of EBF are characteristic of cholecystitis and cholelithiasis: abdominal pain (right upper quadrant and epigastric), nausea, emesis, fatty food intolerance, belching, and flatulence [2].

Diverticular disease is known for fistula complication but a colo-cystic duct fistula is an unknown complication. This case highlights this possibility.

\footnotetext{
Manuscript accepted for publication March 27, 2015

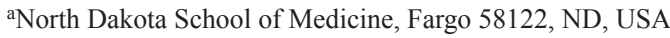

${ }^{\mathrm{b}}$ Caribbean Medical University School of Medicine, Curacao, Chicago Campus, Chicago, IL, USA

${ }^{c}$ Corresponding Author: Lakshmi Kant Pathak, North Dakota School of Medicine, Fargo 58122, ND, USA. Email: drpathaklk@gmail.com
}

doi: http://dx.doi.org/10.14740/jmc2120w

\section{Case Report}

\section{History of present illness}

The patient is an 82-year-old male who presented with four episodes of hematochezia described as dark red blood in the stool. He did not have any nausea, vomiting or abdominal pain. The patient had two similar episodes in the past, one 3 years and another 5 years before the current episode.

\section{Past medical history}

The past medical history included peripheral neuropathy, hypertension, coronary artery disease, bladder cancer, abdominal aortic aneurysm repair and hypothyroidism.

\section{Hospital course}

On admission, his vitals were stable and a physical examination was unremarkable except for a positive stool occult blood test. His complete blood count and electrolytes were within normal limits. CT angiogram of the abdomen showed diverticular disease of the sigmoid colon without diverticulitis. The radiologist also noted air in a structure in the region of the gall bladder and he was not sure if that was volume-averaging with the colon or was related to the gall bladder (Fig. 1). No air was seen in the intrahepatic biliary ducts and there was no evidence of portal venous gas. The patient continued to have bloody bowel movements during his stay in the hospital and he received six units of packed red blood cells. Surgery was on consult and it was decided that the patient should undergo surgery due to the uncontrolled diverticular bleed. At the time of surgery, a diverticulum from the hepatic flexure of the colon was found to be attached to the cystic duct consistent with a fistula between the cystic duct and the hepatic flexure of colon.

The gall bladder showed changes consistent with chronic cholecystitis. Extensive diverticulosis was noted in the left colon. Subtotal colectomy and cholecystectomy were performed. An intraoperative cholangiogram showed a normal common bile duct and normal right and left hepatic ducts with normal drainage into the duodenum. The patient tolerated the proce- 


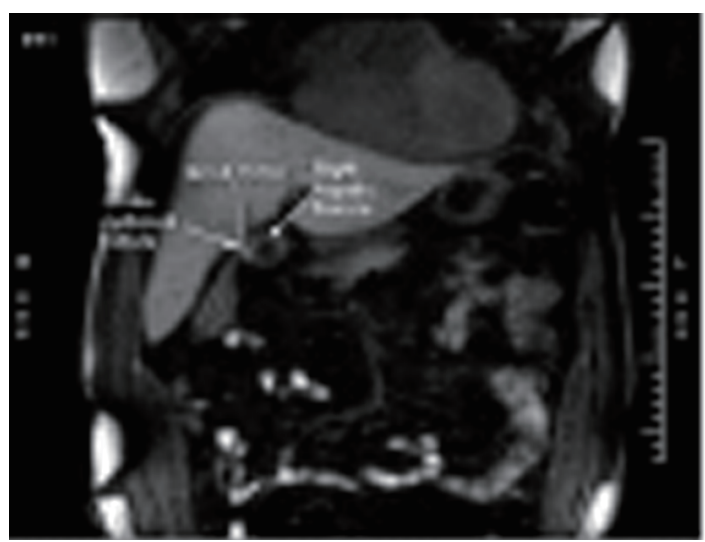

Figure 1. CT scan showing air in the region of cystic duct from colocystic duct fistula.

dure well and left the operating room in stable condition. The recovery was uneventful.

\section{Discussion}

Cholecystocolic fistulae can present with abdominal pain, nausea, weight loss, diarrhea, and dyspeptic symptoms. This fistula can alter the normal enterohepatic circulation of bile acids, leading to their malabsorption. The bile acids also stimulate the colonic mucosa directly to secrete water and electrolytes excessively, leading to steatorrhea and diarrhea. Rare cases have been reported with the stone being impacted at the recto sigmoid causing large bowel obstruction due to such fistulae [1]. If found incidentally during laparotomy, these fistulae should be taken down because of the increased risk of cholecystitis, cholangitis, and a $15 \%$ chance of malignancy in the gall bladder. The most useful techniques for diagnosis are a plain film of the abdomen, abdominal ultrasonography, barium studies, biliary scintigraphy, and ERCP. The standard treatment of a cholecystocolic fistula is open cholecystectomy and closure of the fistula. However, recent developments in laparoscopic surgery have shown its potential use in treating these rare fistulas [2]. Although surgery is the treatment of choice, endoscopic sphincterotomy and CBD stone extraction have been said to cause spontaneous healing of the fistula by reducing increased biliary pressure. This has been used in the treatment of elderly unfit patients [3].

\section{References}

1. Correia MF, Amonkar DP, Nayak SV, Menezes JL. Cholecystocolic fistula: a diagnostic enigma. Saudi J Gastroenterol. 2009;15(1):42-44.

2. Angrisani L, Corcione F, Tartaglia A, Tricarico A, Rendano F, Vincenti R, Lorenzo M, et al. Cholecystoenteric fistula (CF) is not a contraindication for laparoscopic surgery. Surg Endosc. 2001;15(9):1038-1041.

3. Goldberg RI, Phillips RS, Barkin JS. Spontaneous cholecystocolonic fistula treated by endoscopic sphincterotomy. Gastrointest Endosc. 1988;34(1):55-56. 